\title{
An integrated production planning model with obsolescence and lifecycle considerations in a reverse supply chain
}

\author{
Swee S. Kuik, Toshiya Kaihara, Nobutada Fujii, and Daisuke Kokuryo \\ Graduate School of System Informatics, Kobe University, 1-1 Rokkodai, Nada, Kobe Hyogo \\ 657-8501 Japan \\ kuik@kaede.cs.kobe-u.ac.jp, kaihara@kobe-u.ac.jp, nfujii@phoe- \\ nix.kobe-u.ac.jp, kokuryo@port.kobe-u.ac.jp
}

\begin{abstract}
Environmentally conscious manufacturing and product recovery are considered as an important research in the current business scenario. This is due to the associated costs for virgin materials and waste disposal treatment that have been significantly increased in a yearly basis. Currently, there is a lack of modeling into the problems of remanufacturing production planning with component obsolescence and lifecycle constraints. In practice, the planning decisions may have direct impacts on the amount of wastage and disposal along a reverse supply chain. This article proposes an integrated production planning model with component obsolescence and lifecycle considerations, which helps minimise the total associated costs of production, costs of remanufactured products and components inventory holding, ordering costs, and disposal treatment costs. Numerical examples are also presented to demonstrate this production planning problem for remanufactured products using mixed integer programming optimisation. Finally, several contributions of this study and future works are discussed.
\end{abstract}

Keywords: reverse supply chain, remanufacturing, obsolescence

\section{Introduction}

Manufacturers are now aware that product disposal to landfill may not be a viable solution to cope with the increased virgin material and disposal costs [1-3]. In supply chain perspectives, the depreciation due to its value loss for remanufactured products has a great influence on the profitability of manufacturers. Rapid improvement and development in science and technology has also resulted with shorter product lifecycle [4]. An appropriate production planning for shorter lifecycle products can help reduce the inventory holding costs and improve an overall supply chain performance. In addition, the remanufactured products could lose their perceived utility within end-of-life (EOL) stage [1-3]. To address these issues of obsolescence, manufacturers should consider to integrate this critical aspect in the time-period production planning horizon.

In response to the significant changes in environmental rules and regulations, numerous manufacturers are also keen for implementing environmentally conscious

adfa, p. 1, 2015.

(C) Springer-Verlag Berlin Heidelberg 2015 
manufacturing and product recovery with their supply chain partners for achieving significant amount of cost savings as a whole [2,3]. Remanufacturing is currently known as one of the compromising alternatives to meet the stringent environmental challenges. By considering the alternative dispositions for handling EOL products, manufacturers can then minimise significant amount of waste disposal to landfill. There are organisations that are currently working on product recovery operations, such as Caterpillar, HP, Xerox, etc [2]. For successful production planning, manufacturers need to consider the planning horizon with the obsolescence constraints that may directly impact on the depreciation value of remanufactured products.

At present, the development of production planning with obsolescence consideration for manufacturers is still at the budding stage $[1,3]$. A major limitation is the complication of various types of remanufactured products and component obsolescence constraints, where it is subject to the depreciation value over a certain time-period planning horizon. If the obsolescence restriction is exceeded, any excessive inventory for obsolete products and components may need to be disposed entirely [1-3]. However, the total cost associated with the disposal treatment is considered as the financial burdens for manufacturers. The reduction of substantial amount of waste disposal by manufacturers is indeed a need for cost-effective improvement in a reverse supply chain.

In this article, an integrated remanufacturing production planning model is proposed with the considerations of component obsolescence restrictions. This developed model may help manufacturers to minimise overall supply chain costs. Section 2 presents the formulation of mathematical models in remanufacturing production planning problems. To demonstrate the usefulness of the proposed models, Section 3 and 4 present the numerical case examples with results analyses. Finally, the contributions of this study and future work are also discussed.

\section{Mathematical Formulation}

To address the obsolescence issues in production planning, the mixed integer programming (MIP) optimisation models are developed to analyse various scenarios of costeffective production planning. In this study, there are two mathematical models with or without the incorporation of obsolescence issues that are presented for comparisons.

The first model is developed for an analysis to exclude the component obsolescence restrictions. Meanwhile, the second model is developed for an analysis to include component obsolescence restrictions. These optimisation models aim to minimise an overall associated cost of producing remanufactured products for the next planning horizon of $T$. The detailed mathematical formulations are discussed in the following sections. There are some key assumptions for the developed models that are considered in this study. The input parameters with continuous, integer and binary variables used for model formulation and evaluation are provided in Table 1 and 2 respectively.

- Types of the remanufactured products and components are known;

- Demands in the planning horizon are deterministic;

- Capacity in production and ordering are deterministic and constant;

- Quality aspect is excluded for either products or components; 
- Considerations of obsolete components are deterministic and known;

- Transportation and distribution are beyond the scope of this study.

Table 1. Model Parameters

\begin{tabular}{cl}
\hline \hline Symbol & \multicolumn{1}{c}{ Description } \\
\hline$N$ & Number of remanufactured product $i$, where $i=1,2,3, \ldots N$ \\
$J$ & Number of component $j$, where $j=1,2,3, \ldots J$ \\
$T$ & Planning horizon comprising of time period $t$, where $t=1,2,3, \ldots T$ \\
$f$ & Time period $f$, upon receiving component $j$, \\
$R_{i}$ & Unit cost of remanufactured product $i$ \\
$C_{j}$ & Unit cost of component $j$ \\
$U_{i}$ & Inventory holding unit cost for remanufactured product $i$ \\
$H_{j}$ & Inventory holding unit cost for component $j$ \\
$A_{i}$ & Fixed set-up cost for remanufactured product $i$ \\
$G_{j}$ & Fixed ordering cost for component $j$ \\
$V_{i}$ & Production capacity for remanufactured product $i$ \\
$M_{j}$ & Ordering capacity for component $j$ \\
$\alpha_{j}$ & Disposal treatment unit cost for component $j$ \\
$S_{j}$ & Order lot size ordering for component $j$ \\
$O_{j}$ & Order lead-time for component $j$ \\
\hline
\end{tabular}

Table 2. Model Variables

\begin{tabular}{cl}
\hline \hline Symbol & Description \\
\hline$X_{i t}$ & Quantity of i produced in t period \\
$I_{i t}$ & Quantity of inventory $i$ in t period \\
$y_{j t}$ & Quantity of $j$ ordered in t period \\
$L_{j t}$ & Quantity of inventory j in t period \\
$L_{j t f}$ & Quantity of inventory in t period upon receiving $j$ at the end of f period \\
$\beta_{j}$ & Quantity of disposal treatment $j$ \\
$\gamma_{j r t}$ & Quantity of usage in t period upon receiving $j$ at the end off period \\
$D_{i t}$ & Demand $i$ in t period \\
$B_{j i}$ & Bill of Material (BOM) with $j$ of $i$ \\
$e_{j}$ & Obsolescence with $j$ lifecycle \\
$a_{i t}$ & Fixed setup-up of $i$ in t period (binary) \\
$P_{j t}$ & Fixed (scheduled) ordering of $j$ in t period (binary) \\
$p_{j t}$ & Fixed ordering of $j$ in t period (binary) \\
&
\end{tabular}




\subsection{Model One without Component Obsolescence and Lifecycle Constraints}

This section presents the formulation of MIP model to evaluate production planning problem by excluding component obsolescence constraint. Using the above parameters and decision variables from Table 1 and 2, the objective function of MIP model is formulated into eight terms as shown in Eq. (1).

$$
\begin{aligned}
\operatorname{Min} Z=\sum_{i} \sum_{t} & R_{i} X_{i t}+\sum_{j} \sum_{t} C_{j} y_{j t}+\sum_{j} \sum_{t} C_{j} Y_{j t} \\
& +\sum_{i} \sum_{t} U_{i} I_{i t}+\sum_{i} \sum_{t} A_{i} a_{i t}+\sum_{j} \sum_{t} G_{j} P_{j t} \\
& +\sum_{j} \sum_{t} G_{j} p_{j t}+\sum_{j} \sum_{t} H_{j} L_{j t}
\end{aligned}
$$

Terms 1-3 are the cost associated with product-component in production and inventory aspects. Term 5 is calculated as the set-up associated costs in $t$ period planning horizon. Terms 6 and 7 represent the cost associated with ordering components. Term 8 is the inventory cost associated with component in $t$ period planning horizon. The model constraints are presented in Eqs. (2)-(8).

$$
\begin{array}{lcr}
L_{j t}=L_{j, t-1}+S_{j} Y_{j t}-\sum_{i} B_{j i} X_{i t} & 1<t \leq o_{j} & \forall t, j \\
L_{j t}=L_{j, t-1}+S_{j} y_{j, t-o_{j}}-\sum_{i} B_{j i} X_{i t} & t>o_{j} & \forall t, j \\
I_{i t}=I_{i, t-1}+X_{i t}-D_{i t} & & \forall t, j \\
X_{i t} \leq V_{i} \quad y_{j t} \leq M_{j} & & \forall t, j \\
y_{j t} \leq W P_{j t} \leq W a_{i t} & X_{i t} \leq W p_{j t} & \forall t, i \\
X_{i t}, y_{j t}, Y_{j t}, I_{i t}, L_{j t} \geq 0 & & \forall t, i, j, f
\end{array}
$$

Eq. (2) is the inventory balance associated with order and bill of materials (BOM). Eqs. (3) and (4) are the formulation of remanufactured products for the inventory balance to satisfy demand request. The constraints with related capacity, such as production and ordering are shown in Eq. (5). Meanwhile, Eq. (6) is formulated as the parameters used in binary form, if the values for $y_{j t}, X_{i t}$, and $Y_{j t}$, and $p_{j t}$ are more than zero, $P_{j t}, a_{i t}$, and $p_{j t}$ are also more than zero. These values of $y_{j t}, P_{j t}$, and $p_{j t}$ can be expressed as one with $W$ as a large positive number. In addition, Eqs. (7) and (8) represent the integer and binary decision variables used in the modelling.

\subsection{Model Two with Component Obsolescence and Lifecycle Constraints}

This section presents the formulation of MIP model to evaluate production planning with the restriction of component obsolescence. An objective function is formulated 
with nine terms as shown in Eq. (9), where Eqs. (10)-(25) are the constraints used for remanufacturing production modelling in this study.

$$
\begin{aligned}
\operatorname{Min} Z=\sum_{i} \sum_{t} & R_{i} X_{i t}+\sum_{j} \sum_{t} C_{j} y_{j t}+\sum_{j} \sum_{t} C_{j} Y_{j t}+\sum_{i} \sum_{t} U_{i} I_{i t} \\
& +\sum_{i} \sum_{t} A_{i} a_{i t}+\sum_{j} \sum_{t} G_{j} P_{j t} \\
& +\sum_{j} \sum_{t} G_{j} p_{j t}+\sum_{j} \sum_{t} \sum_{f} H_{j} L_{j t f}+\sum_{j} \alpha_{j} \beta_{j}
\end{aligned}
$$

There are only two modified terms with this proposed model. Term 8 is modified to include the time-period upon receiving components and the end of obsolete timeperiod. The component disposal treatment cost is calculated as shown in the Term 9. The rest of the Terms 1- 7 used are similar with the previous model in Eq. (1).

$$
\begin{aligned}
& I_{i t}=I_{i, t-1}+X_{i t}-D_{i t} \quad \forall t, j \\
& \sum_{f}^{t} L_{j t f}=L_{j 0}+S_{j} Y_{j t}-\sum_{i} B_{j i} X_{i t} \quad t=1 \quad \forall t, j, r \\
& \sum_{f}^{t} L_{j t f}=\sum_{f}^{t-1} L_{j, t-1, f}+S_{j} Y_{j t}-\sum_{i} B_{j i} X_{i t} \quad 1<t<e_{j} \quad t \geq e_{j} \quad \forall t, j \\
& \sum_{f=t+1-e_{j}}^{t} L_{j t f}=\sum_{f=t+1-e_{j}}^{t-1} L_{j, t-1, f}+S_{j} Y_{j t} \quad t \geq e_{j} \quad t \geq o_{j} \quad \forall t, j \\
& -\sum_{i} B_{j i} X_{i t} \\
& \sum_{f}^{t} L_{j t f}=\sum_{f}^{t-1} L_{j, t-1, f}+S_{j} y_{j t}-\sum_{i} B_{j i} X_{i t} \quad 1<t<e_{j} \quad t \geq o_{j} \quad \forall t, j \\
& \sum_{f=t+1-e_{j}}^{t} L_{j t f}=\sum_{f=t+1-e_{j}}^{t-1} L_{j, t-1, f}+S_{j} y_{j t} \quad t \geq e_{j} \quad t \geq o_{j} \quad \forall t, j \\
& -\sum_{i} B_{j i} X_{i t} \\
& \sum_{i} B_{j i} X_{i t}=\sum_{f} \gamma_{j f \mathrm{t}} \quad 1 \leq t<e_{j} \quad \forall t, j, r \\
& \sum_{i} B_{j i} X_{i t}=\sum_{f=t+1-e_{j}} \gamma_{j f t} \quad e_{j} \leq t<T \quad \forall t, j, r \\
& L_{j t f}=S_{j} Y_{j t}-\gamma_{j f t} \quad f=t \quad t \leq o_{j} \quad \forall t, j, r \\
& L_{j t f}=S_{j} y_{j t}-\gamma_{j f t} \quad f=t \quad t \leq o_{j} \quad \forall t, j, r \\
& L_{j t f}=L_{j, t-1, f}-\gamma_{j f t} \quad f<t \quad t-f \leq o_{j} \quad \forall t, j, f
\end{aligned}
$$




$$
\begin{aligned}
& \beta_{j}=\sum_{t=o_{j}-1} \gamma_{j, t, t+1-o_{j}} \quad \forall t, j \\
& X_{i t} \leq V_{i} \quad y_{j t} \leq M_{j} \quad \forall t, j \\
& y_{j t} \leq W P_{j t} \quad X_{i t} \leq W a_{i t} \quad Y_{j t} \leq W p_{j t} \quad \forall t, i \\
& X_{i t}, y_{j t}, Y_{j t}, I_{i t}, L_{j t f}, \gamma_{j f t}, \beta_{j} \geq 0 \quad \forall t, i, j, f \\
& a_{i t}, P_{j t}, p_{j t} \in(0,1)
\end{aligned}
$$

Eq. (10) is the balance of inventory holding for product-component to satisfy demand request. Eqs (11) - (15) are calculated as each time-period of orders is met with scheduled production planning. Eqs. (16) and (17) represent the production usage at each time-period with the obsolete components. Eqs. (18) - (20) are the expressions of inventory holding for product-component at each time-period. Eq. (21) is derived to estimate excessive components to be discarded at the end of the obsolete time-period. In addition, the capacity constraints in relation to the production and ordering are established as shown in Eq. (22). Eq. (23) represents the decision variable in binary form. If the values of $y_{j t}, X_{i t}, Y_{j t}$, and $p_{j t}$ are more than zero, these variables of $P_{j t}, a_{i t}$, and $p_{j t}$ are also more than zero. As a result, these variables of $y_{j t}, P_{j t}$, and $p_{j t}$ are one, where $W$ is a large positive number.

Finally, Eqs. (24) and (25) represent the continuous and binary decision variables used in the modelling. In order to resolve the developed optimization models with obsolete component scenario in Eqs. (1) and (9), a CPLEX mixed integer optimization solver is used.

\section{$3 \quad$ Numerical Example}

This section discusses a numerical case example for production planning problem with the remanufactured products. In this example, a planning horizon is about 12 periods, which is used as a simulated case example. In this remanufacturing production planning example, there are three types (i.e. PT1, PT2 and PT3) of remanufactured product under examination. Each type of the remanufactured products has four separated components, which is named as CP1, CP2, CP3 and CP4. There are considered as the common components that are used by all types of the remanufactured products. Table 3 and 4 show that the parameters used for both remanufactured products and components in the modelling. The obsolescence of time-period estimation for each component is about 6 months for $\mathrm{CP} 1,3$ months for $\mathrm{CP} 2,9$ months for $\mathrm{CP} 3$, and 3 months for CP4 respectively.

Using Cplex optimisation solver, the results obtained without obsolescence issue showed that an overall minimum cost for the production planning was approximately $\$ 5,263,143$ with the linear relaxation of approximately $\$ 4,829,131$. The calculated value of optimality gap is about 0.0825 . However, the results obtained with obsolescence issues revealed that an overall minimum cost for production planning was approximately $\$ 6,029,983$ with the linear relaxation of approximately $\$ 5,528,876$. The 
calculated value of optimality gap is about 0.0906 . By comparing both models with or without obsolescence, the obtained results revealed that the total cost for production planning model with obsolescence as derived in Eq. (9) would be much higher than the total cost for production planning model without obsolescence as derived in Eq. (1). One of the primary reasons is that the product-component inventory holding and disposal are expressed as the cost associated terms of objective functions. In practice, the manufacturer should consider to maintain inventory stocks with quantities of the obsolete components as low as possible to avoid disposal costs after EOL constraints.

Table 3. Parameters used product $i$ for modelling

\begin{tabular}{|c|c|c|c|}
\hline & PT1 & PT2 & PT3 \\
\hline$V_{i}$ & 300 & 700 & 700 \\
\hline$R_{i}$ & 115 & 100 & 230 \\
\hline$U_{i}$ & 56 & 48 & 77 \\
\hline$A_{i}$ & 530 & 780 & 840 \\
\hline
\end{tabular}

Table 4. Parameters used component $j$ for modelling

\begin{tabular}{|c|c|c|c|c|}
\hline & $\boldsymbol{C P 1}$ & $\boldsymbol{C P 2}$ & $\boldsymbol{C P 3}$ & $\boldsymbol{C P 4}$ \\
\hline$S_{j}$ & 4500 & 4500 & 10000 & 10000 \\
\hline$M_{j}$ & 60 & 30 & 6 & 8 \\
\hline$C_{j}$ & 7500 & 22000 & 35000 & 23000 \\
\hline$L_{j t}$ & 4 & 5 & 5 & 3 \\
\hline$\alpha_{j}$ & 6 & 8 & 9 & 6 \\
\hline$G_{j}$ & 420 & 540 & 680 & 460 \\
\hline$O_{j}$ & 30000 & 22000 & 5000 & 0 \\
\hline
\end{tabular}

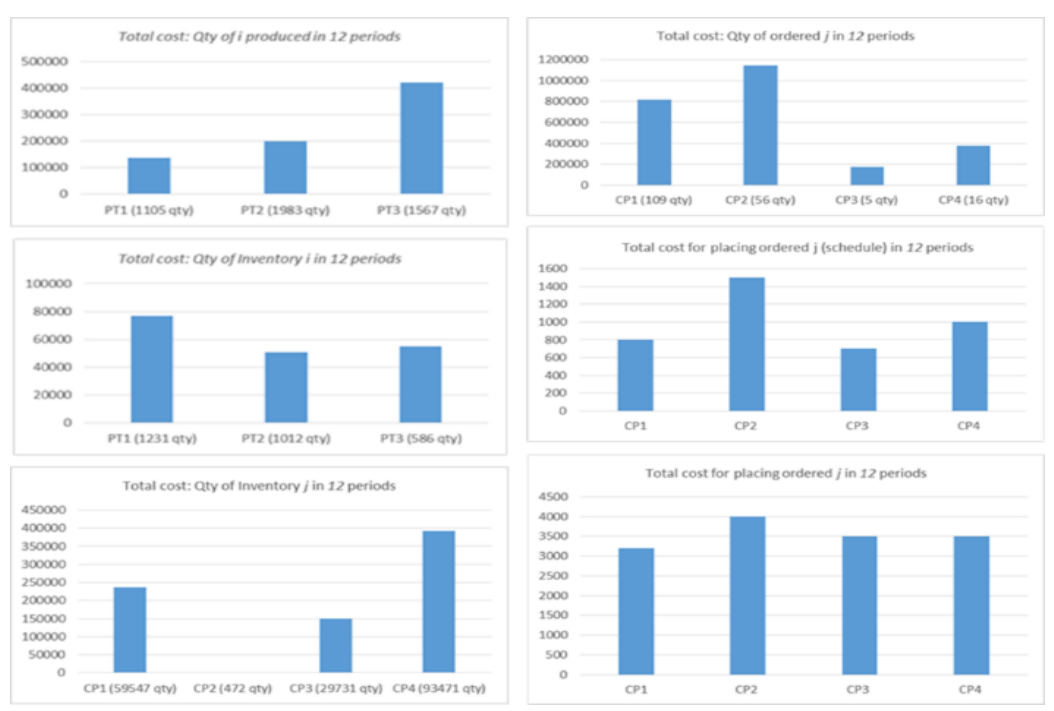

Fig. 1. A summary of total associated costs for producing 3 product types with 4 components 


\section{Results and Discussions}

The obsolescence issues for remanufacturing production planning has been drawn significant attentions in recent years. To minimise the costs of producing the remanufactured products, there is a need to consider the obsolescence issues for product-component level in a production planning horizon. Figure 1 illustrates a summary of product and component inventory variables with the total associated costs for this case example. This consideration may help resolve the oversimplified production planning problems. Two developed models have been compared with the remanufacturing production planning with and without obsolescence considerations. With three remanufactured product types $(N=3)$ and four separate components $(J=4)$, the proposed MIP model with obsolescence scenario consists of 1,107 variables with binary variables of 132, integer variable of 970 and continuous variable of 5. This model contains 2392 constraints used and non-zero coefficient of 1968.

\section{$5 \quad$ Concluding Remarks}

In this study, the proposed production planning model is developed by integrating the obsolescence issues for remanufactured products within a planning horizon. Comparisons of the obtained results from the developed models with and without obsolescence showed that the total associated costs with obsolescence are generally higher than the total associated costs without obsolescence. In addition, the contribution of this study is twofold. Firstly, the developed model addresses the obsolescence issue for remanufactured products in production planning problems. Secondly, the developed model aims to avoid the oversimplification of cost estimation in a reverse supply chain. For future works, different obsolete time-periods for the remanufactured product-component and variations of the inventory holding costs in production planning will be compared. Furthermore, the models can be extended by considering uncertainty of the consumer's demand requirements.

\section{Reference}

1. Pahl, J. and S. Voß, Integrating deterioration and lifetime constraints in production and supply chain planning: A survey. European Journal of Operational Research, 2014. 238(3): p. 654-674.

2. Kuik, S.S., S. Nagalingam, and Y. Amer, Sustainable supply chain for collaborative manufacturing. Journal of Manufacturing Technology Management, 2011. 22(8): p. 984-1001.

3. Nagalingam, S.V., S.S. Kuik, and Y. Amer, Performance measurement of product returns with recovery for sustainable manufacturing. Robotics and Computer-Integrated Manufacturing, 2013. 29(6): p. 473-483.

4. Zhao, Y., et al., Varying Lifecycle Lengths Within a Product Take-Back Portfolio. Journal of Mechanical Design, 2010. 132(9): p. 091012-091012. 